\title{
Studi per la conservazione e la valorizzazione del Patrimonio culturale e del paesaggio di Tivoli
}

\author{
Antonio Pugliano \\ Università di Roma Tre \\ antonio.pugliano@uniroma3.it
}

RIASSUNTO: II saggio descrive l'iniziativa scientifica per valorizzare il patrimonio culturale e il paesaggio di Tivoli, antica città del Lazio appartenente all'Area Metropolitana di Roma, al fine di creare un ambiente adatto alla migliore qualità della vita in città e per la tutela dei suoi monumenti. A tal fine è stata analizzata la struttura urbana di Tivoli e dei suoi edifici storici. Gli studi esplorano le potenzialità di materiali da costruzione tradizionali e metodi, usati nell'habitat vernacolare e producono «forme organizzate di conoscenza» utili a sostenere sia le nuove iniziative di trasformazione urbana ed edilizia, sia le future attività di governo della città. La conoscenza storica del sito è organizzata in un insieme strutturato: i dati relativi alla documentazione dei processi di trasformazione storica del tessuto urbano e di monumenti architettonici e archeologici sono raccolti in un sistema di gestione delle informazioni digitali (GIS).

PAROLE CHIAVE: Tivoli, Conservazione, Valorizzazione, GIS, Patrimonio culturale, Paesaggio.

\section{Studies for Preservation and Enhancement of Cultural Heritage and Landscape of Tivoli}

ABSTRACT: The paper describes the scientific initiative to enhance the cultural heritage and the landscape of Tivoli, an ancient city of Latium belonging to Area Metropolitana di Roma, in order to create an environment suitable for the best quality of city life and for the protection of its monuments. To this end the urban structure of Tivoli and its historic buildings were analyzed. Studies explore the potential of traditional building materials and methods used in the vernacular habitat and produce «organized forms of knowledge» useful for supporting both the new urban and building transformation initiatives, and the future government activities in the city. Historical knowledge of the site is organized in a structured set: the data relating to the documentation of historical transformation processes of urban texture and of architectural and archaeological monuments are collected in a digital information management system (GIS).

KEYWORDS: Tivoli, Preservation, Enhancement, GIS, Cultural Heritage, Landscape.

Recibido: 14 de abril de 2016 / Aceptado: 31 de mayo de 2016.

\section{Premessa normativa: il Patrimonio Culturale come Eredità}

Il concetto di patrimonio inteso come eredità ha un'origine antica, nella lingua latina: patrimonium, deriva da pater, «padre», e munus, «ufficio»; il termine individua la «funzione del padre» cioè l'attività di governo, fatta di diritti e di obblighi, che è svolta da quest'ultimo nell'interesse di una comunità familiare che a esso è riferita. A vantaggio di quest'ultima, pertanto, la funzione si esercita per generare, conservare e accrescere la consistenza economica ovvero la disponibilità di risorse strumentalmente utili a soddisfare il novero dei bisogni umani, tanto materiali quanto spirituali. Con il patrimonio s'identifica, quindi, la ricchezza di un singolo o di una comunità in un determinato momento; esso rappresenta una ricchezza solida, una risorsa, idonea a generare la dinamica reddituale attraverso il suo impegno, in forme appropriate, all'interno di processi economici durevoli nel

PUGLIANO, Antonio: "Studi per la conservazione e la valorizzazione del Patrimonio culturale e del paesaggio di Tivoli», Boletín de Arte, n. ${ }^{\circ} 37$, Departamento de Historia del Arte, Universidad de Málaga, 2016, pp. 157-174, ISSN: 0211-8483. 
tempo che non comportino il consumo o la perdita del suo valore intrinseco.

\section{Il concetto di patrimonio nel Codice italiano dei Beni Culturali}

Nel panorama legislativo attuale italiano in materia di Beni Culturali il patrimonio è definito in una forma che può apparire riduttiva, essendo riferita esclusivamente alla consistenza materiale e non alla sua genesi. II Codice dei Beni Culturali recita: «॥ patrimonio culturale è costituito dai beni culturali e dai beni paesaggistici. Sono beni culturali le cose immobili e mobili che [...] presentano interesse artistico, storico, archeologico, etnoantropologico, archivistico e bibliografico e le altre cose individuate dalla legge o in base alla legge quali testimonianze aventi valore di civiltà. Sono beni paesaggistici gli immobili e le aree [...] costituenti espressione dei valori storici, culturali, naturali, morfologici ed estetici del territorio, e gli altri beni individuati dalla legge o in base alla legge. I beni del patrimonio culturale di appartenenza pubblica sono destinati alla fruizione della collettività, compatibilmente con le esigenze di uso istituzionale e sempre che non vi ostino ragioni di tutela.»1

I concetti di «eredità culturale», «paesaggio» e «comunità di eredità»

Le elaborazioni concettuali espresse dalla Convenzione Europea del Paesaggio ${ }^{2}$ e dalle Convenzioni a essa precedenti ${ }^{3}$ hanno introdotto, invece, un concetto estensivo di patrimonio, molto prossimo a quello suggerito dalla etimologia del vocabolo; come vedremo più avanti detto concetto, articolato e reso fertile di interpretazioni operative, orienta i contenuti della successiva Convenzione di Faro ${ }^{4}$.

Nella traduzione italiana della Convenzione di Faro curata dal Ministero per i Beni e le Attività Culturali e per il Turismo, l'espressione «Cultural Heritage» è stata intenzionalmente tradotta con «eredità culturale». La definizione scelta serve ad affermare che il patrimonio è un insieme composito di beni che ha come carattere fondamentale il fatto di essere generato dalla sedimentazione di azioni e prodotti di comunità precedenti che viene acquisito dalle comunità successive per mezzo del trasferimento legittimo da parte dei predecessori.
Tale definizione è utile a introdurre l'idea che la gestione di un bene ereditario impone un impegno di carattere profondamente morale ai suoi fruitori che sono chiamati a svolgere il ruolo transitorio di amministratori di una «eredità culturale usata saggiamente come risorsa per lo sviluppo sostenibile e per la qualità della vita, in una società in costante evoluzione» ${ }^{5}$. L'eredità culturale, pertanto, identifica un selezionato lascito materiale e immateriale del passato nel quale le popolazioni «indipendentemente da chi ne detenga la proprietà» identificano l'immagine emblematica e l'espressione identitaria «di valori, credenze, conoscenze e tradizioni, in continua evoluzione» 6 .

Nell'eredità culturale convergono tutte le componenti di valore e significato riconoscibili nell'ambiente di vita come il risultato dell'interazione, avvenuta nel volgere del tempo, fra le popolazioni e i luoghi da esse abitati; tale interazione è al centro anche della definizione del concetto attuale di Paesaggio, maturato in Italia a partire dalla Legge Cro$\mathrm{ce}^{7}$ e delineato compiutamente nella Convenzione Europea del Paesaggio, considerando l'equilibrio tra i contributi delle componenti storica, estetica e naturale al processo di identificazione del territorio in Paesaggio culturale da parte della comunità [1]. La Convenzione Europea del Paesaggio compone quindi le premesse alla Convenzione di Faro individuando l'ambito concreto cui riferire le iniziative legate alla gestione del valore che l'eredità culturale offre alla società. Entrambe le Convenzioni considerano la qualità dell'ambiente di vita che va assicurata attraverso trasformazioni adattative e partecipate, riconoscendo alla comunità il ruolo principale di governo (Maniglio Calcagno, 2015). Lo strumento operativo, nel concreto, per il controllo delle fisiologiche mutazioni del territorio è la «pianificazione» che dovrà concepire, proporre e sviluppare «azioni fortemente lungimiranti, volte alla valorizzazione, al ripristino o alla creazione di paesaggi».

Il paesaggio pianificato è, quindi, il museo della stratificazione culturale e civile di una comunità; esso compone l'ambito di esistenza del patrimonio e lo contiene: non s'identifica semplicemente con il territorio ma con quella parte di esso che è percepita dalla comunità come l'esito significante di processi d'integrazione tra la natura e l'uomo, determinati da quest'ultimo attraverso le sue attività pertinenti alle dimensioni storica, materiale, culturale.

II paesaggio, pertanto, si compone della sua realtà materiale e della sua realtà percepita, ne consegue che il 
1. II concetto moderno di paesaggio informa la composizione de La Tempesta, dipinto nel quale Giorgione riunisce in una unica rappresentazione di sintesi la natura, severa, le attività e le realizzazioni dell'uomo, la storia cui alludono le vestigia archeologiche (Gombrich, 1977). L'integrazione tra queste componenti, materiali e immateriali, prende la forma di un paesaggio che assume al suo interno la componente umana e diviene il protagonista del dipinto: da questo momento il paesaggio, come metafora dell'ambiente di vita, tende a estendere

la sua pertinenza dalla rappresentazione alla interpretazione della realtà e a divenire lo strumento della comunicazione di valori da comprendere e condividere. GIORGIONE, La Tempesta (1505-8). Venezia. Gallerie dell'Accademia

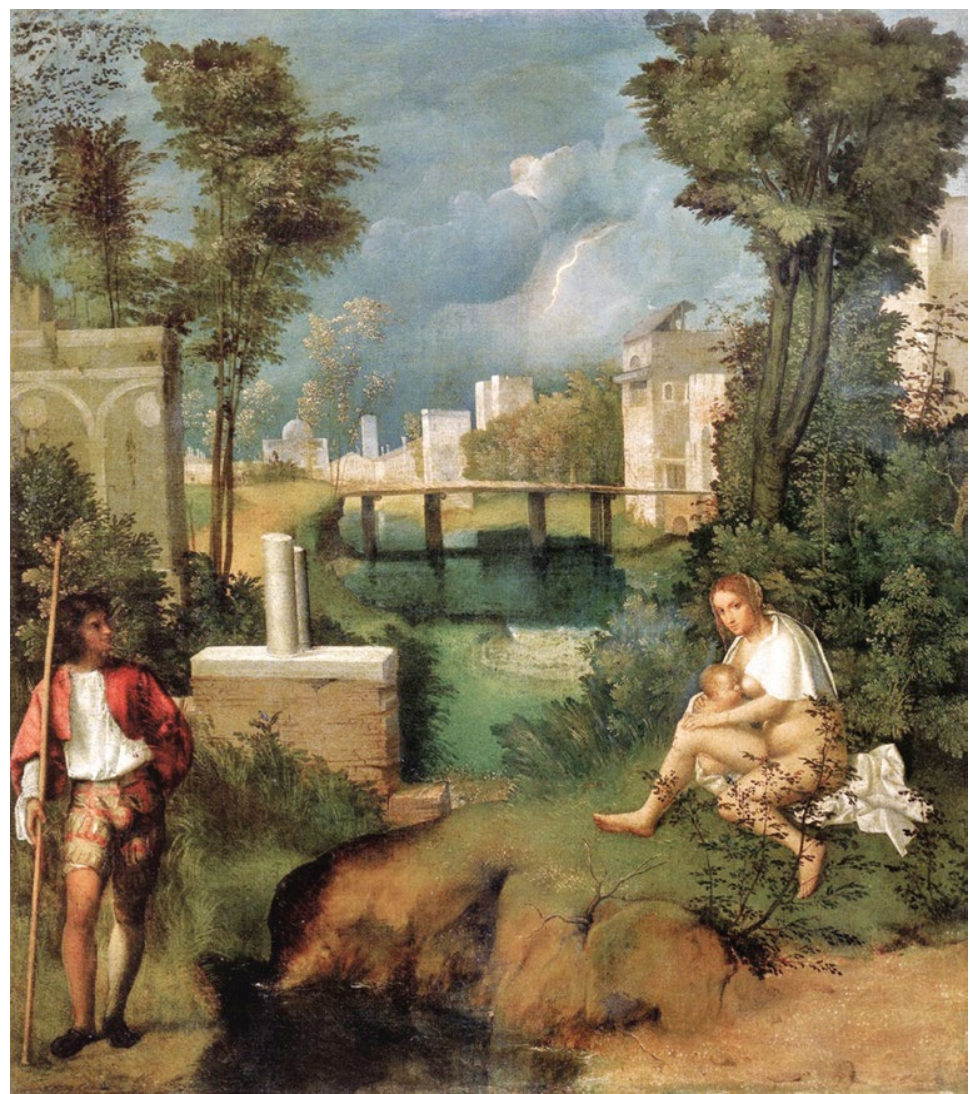

paesaggio è tale se a esso la comunità riconosce un ruolo identitario dal quale è possibile derivare il valore di emblematicità [2].

Le componenti materiali -la natura pura o artefatta, l'architettura, l'uomo- e immateriali -la storia- sono inseparabili e si integrano imponendo un analogo sforzo di integrazione al fruitore del paesaggio, chiamato a coniugare all'apprezzamento estetico la comprensione del valore culturale e la condivisione del significato [3]. Le popolazioni inclini a riconoscersi nel quadro identitario espresso dall'eredità culturale, nel suo contesto ambientale, costituiscono la «comunità di eredità»; a essa spetta l'onere della sua conservazione, implemento e trasmissione alle generazioni future.

Secondo le indicazioni della Convenzione, nel quadro di un'azione pubblica, la comunità deve porre in essere attività programmatiche e progettuali, complete di «valutazioni di impatto» utili a rendere adattative le trasformazioni necessarie all'ambiente di vita. Si tratta di promuovere lo sviluppo sostenibile, la diversità culturale e la creatività contemporanea attraverso strategie integrate che siano basate sull'implemento e la diffusione della conoscenza all'interno di iniziative educative e di formazione permanente anche di carattere professionale ${ }^{9}$.

\section{Conservazione attiva e sostenibilità. II linguaggio} architettonico come fattore identitario delle culture locali

L'uso sostenibile dell'eredità culturale deriva dall'esercizio di una progettualità attenta alla protezione e al recupero delle espressioni della cultura materiale locale attraverso il primato della manutenzione sul rinnovo e la sostituzione di artefatti e contesti ambientali stratificati storicamente. Tali espressioni materiali della tradizione, come del resto la struttura e i lemmi di una lingua parlata, sono il mezzo di diffusione 


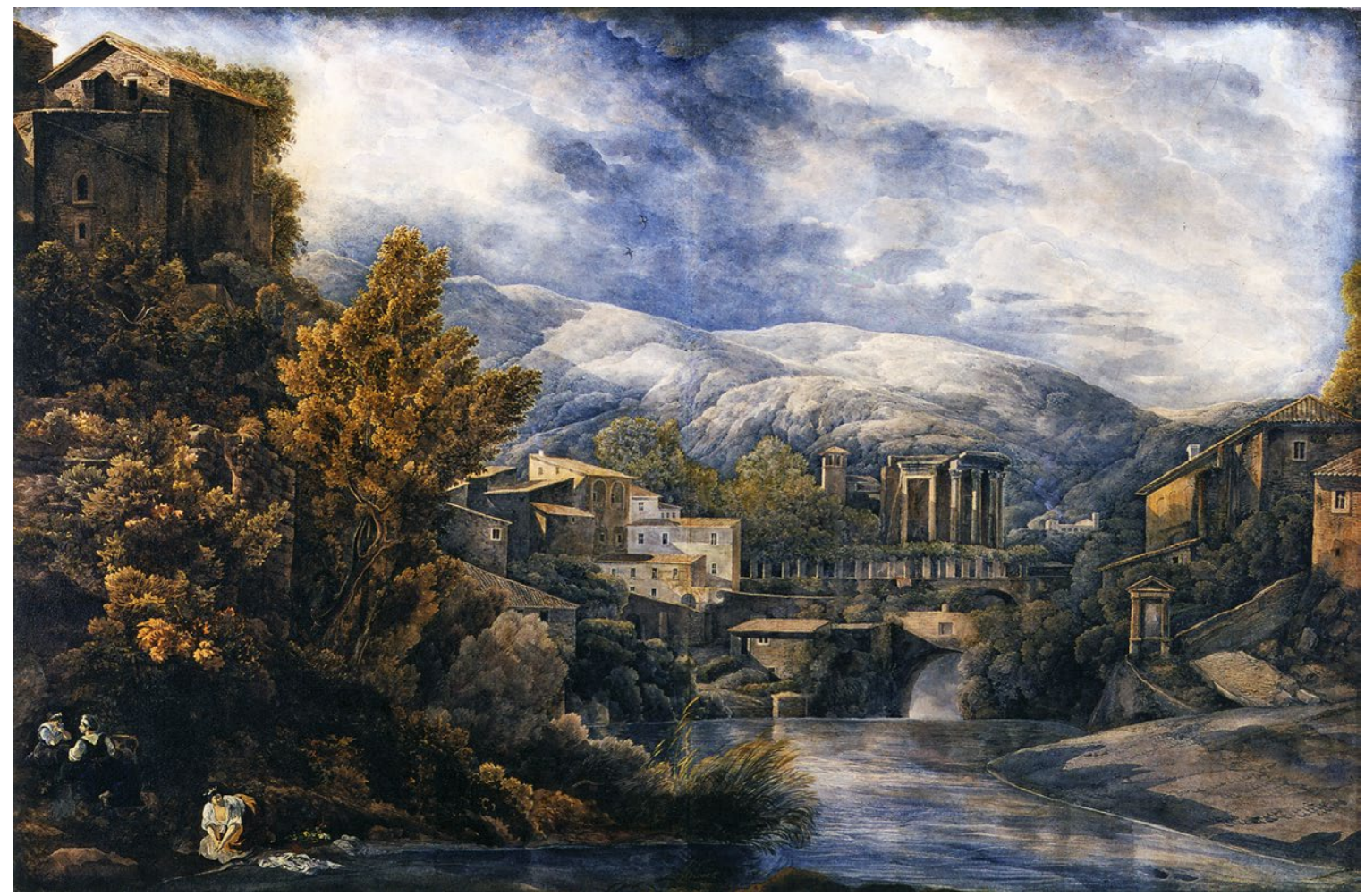

2. L'acqua è una componente essenziale, di primo piano, nel paesaggio tiburtino: l'Aniene è il fulcro delle attività umane ed è la genesi millenaria dell'insediamento cittadino; per questo l'Aniene è vicino alla città, più di quanto lo sia nella percezione della realtà fisica, come suggerisce questo dipinto che, per la scelta di un punto di vista affatto banale (vista da monte della cascata) costruisce una singolare analogia con il tranquillo e solenne paesaggio del Tevere a Roma, nell'area del foro boario. DUCROS Abraham Louis Rodolphe, Vue de Tivoli et du Temple de la Sibylle (1785). Losanna, Musée cantonal des Beaux-Arts, Collezione Ducros (Cogotti, 2014: 50)

di dati culturali, antropologici e storici, importanti e non altrimenti riconoscibili. Pertanto, nel restauro e nel recupero, cioè nell'esercizio delle attività progettuali deputate ad attuare la pratica della conservazione e della valorizzazione, è opportuno riconsiderare i magisteri della tradizione e l'uso di materiali e tecniche consolidati nella storia; il medesimo atteggiamento può essere ritenuto auspicabile nella prospettiva di sperimentare il potenziale applicativo della cultura materiale locale anche in occasione delle realizzazioni contemporanee ${ }^{10}$. Nella sostanza, si tratta di costruire e diffondere una nuova cultura della progettazione architettonica e del restauro che sia critica, storicamente consapevole e filologicamente competente.

L'attività di formazione della comunità alla fruizione e al governo dell'eredità culturale comporta, quindi, la realizza- zione d'idonei supporti conoscitivi e metodologici. La Convenzione di Faro pone in evidenza le necessità di sviluppare modelli di comportamento standard per lo studio, la documentazione e il riconoscimento di valore antropologico, storico, artistico dei contesti ambientali e suggerisce di definire «buone prassi», codificandole e garantendone la diffusione affinché sostengano la progettazione degli interventi necessari alla loro conservazione e valorizzazione.

\section{Thesauri di Architettura e Manuali del recupero}

Gli strumenti necessari allo sviluppo di programmi di educazione al patrimonio sono, quindi, raccolte sistematiche di fonti per il riconoscimento del valore storico e antropologico 

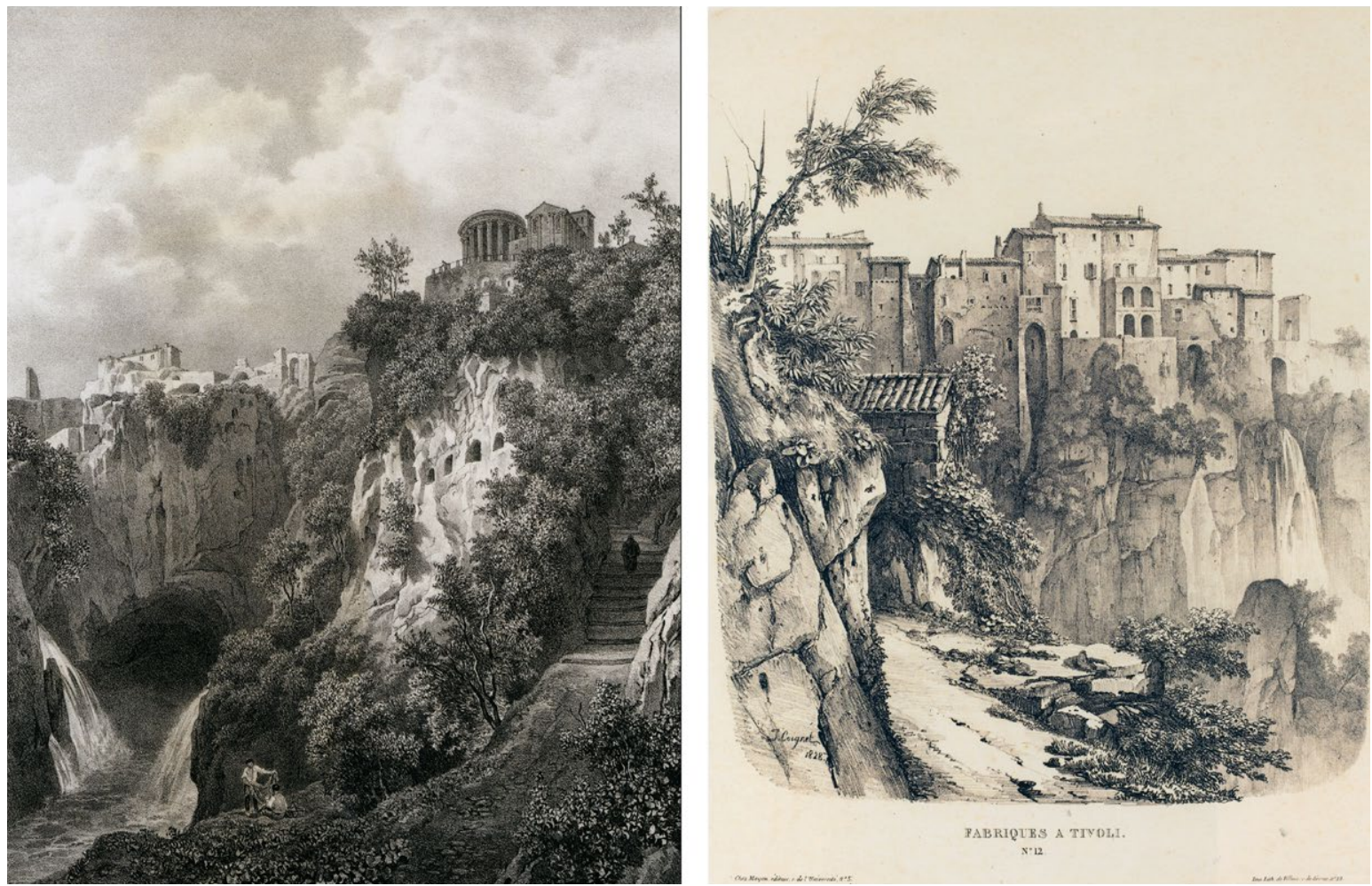

3. II Paesaggio antropico di Tivoli è ricco di suggestioni che riguardano tanto la percezione della storia del luogo, dai trascorsi mitici (a sinistra), quanto la manifestazione tangibile dello sforzo incessante dell'uomo di governare l'ambiente naturale per sovrastarlo e adattarlo ad accogliere le necessità del vivere quotidiano (a destra). DEROY Isidore Laurent, Veduta panoramica dal basso della città di Tivoli e dell'Acropoli tiburtina con i suoi templi. (1860 c.a.) Tivoli, Villa d'Este, Collezione Lemmerman (a sinistra). COIGNET Jules, Fabriques à Tivoli (1828), Washington, National Gallery of Art. (a destra) (Cogotti, 2014: 54 e 102)

del territorio e degli artefatti che lo compongono e sintesi di genere manualistico utili ad informare la pratica del restauro. Si tratta di documentare e analizzare le espressioni caratteristiche e ricorrenti nella costruzione dell'ambiente antropizzato attraverso le quali diffondere la conoscenza storica dei processi culturali e sociali, formativi e trasformativi. Gli argomenti centrali della trattazione sono i modi dell'abitare e del costruire, esposti attraverso la definizione del loro sistema di relazioni reciproche, motivate storicamente, che determinano la fisionomia concettuale di percorsi territoriali e urbani, d'insediamenti, di architetture e componenti [4]. Da questa conoscenza discende il repertorio di scelte tecniche di restauro da sviluppare in occasione dell'intervento di valorizzazione, cioè di conservazione attiva e sostenibile, del patrimonio e del paesaggio che lo ospita. II metodo restaurativo cui si fa riferimento è filologico ovvero ricondu- cibile a «quell'onorevole arte che esige dal suo cultore di trarsi in disparte, lasciarsi tempo, divenire silenzioso, divenire lento, in quanto è un'arte e una competenza di orafi della parola, che deve compiere soltanto lavori finissimi che richiedono cautela [...] essa insegna a leggere bene, cioè lentamente, profondamente, con riguardo e precauzione, con pensieri reconditi, lasciando porte aperte, con dita e occhi delicati...» (Nietzsche, 1992: 8-9). Tale orientamento operativo è interessante poiché genera sintesi culturali inedite e attuali e consente di superare consapevolmente la tendenza elementare all'esercizio della tutela in una chiave passiva che, seppure adatta alla conservazione di contesti integri, leggibili e in ottime condizioni di esercizio, appare palesemente l'inefficace ai fini della valorizzazione del senso e della consistenza di ambiti del territorio degradati, malintesi e sottoutilizzati. 


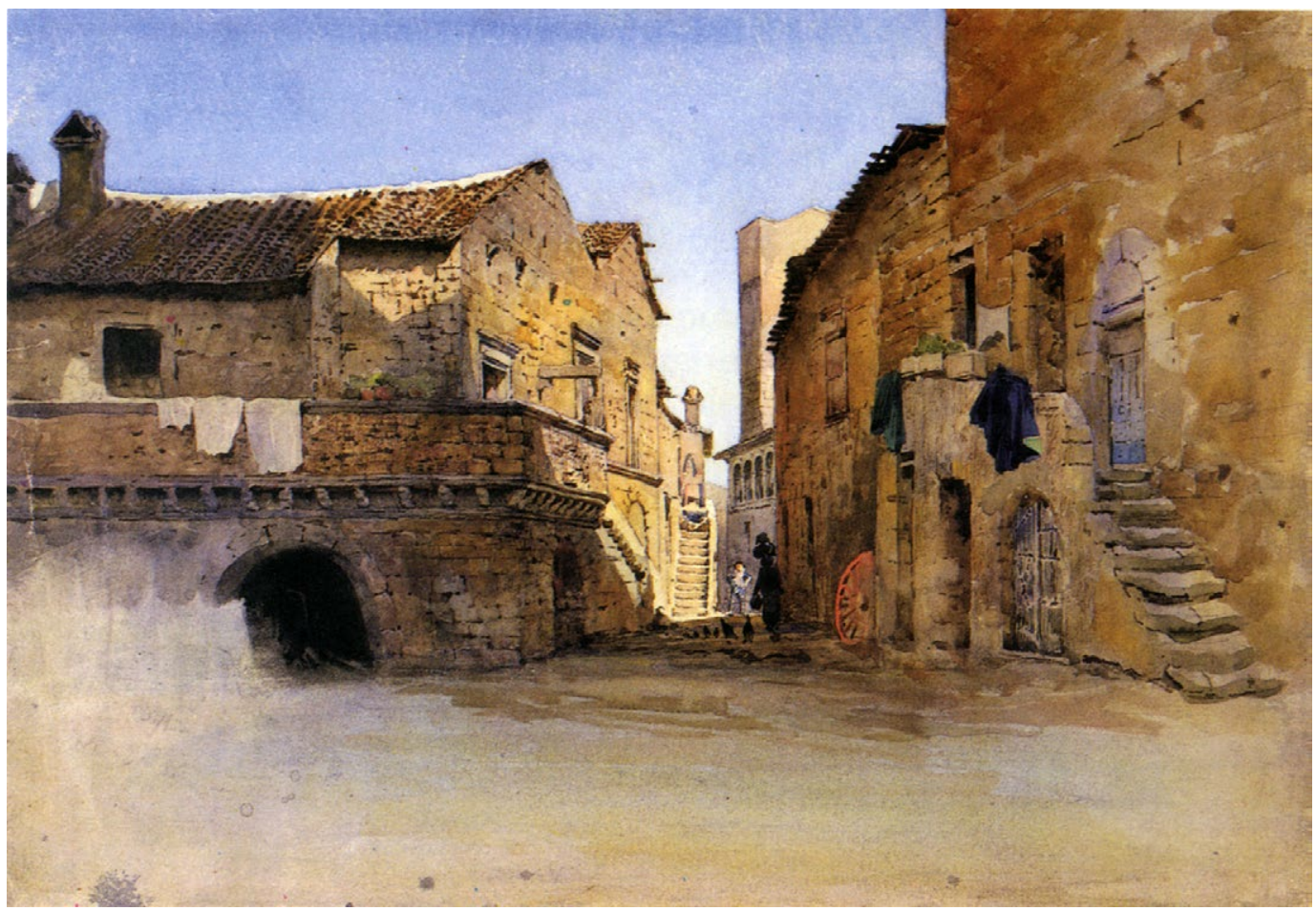

4. II paesaggio urbano di Tivoli si compone di architetture profondamente stratificate che conservano integro il carattere dell'ambiente urbano storico. L'immagine documenta la sopravvivenza di un tipo edilizio del medioevo, residenziale, dalla decorazione peculiare, molto diffuso in Tivoli: la casa a profferlo, con scala di accesso all'esterno. ROESLER FRANZ, Ettore. Vicolo del Lavatoio (1906) (Cogotti, 2014: 105)

Il linguaggio per la comunicazione della cultura materiale.

\section{Disegno e storiografia}

La matrice comune di tali elaborazioni è il disegno, in una dimensione interscalare che va dalla cartografia territoriale alla rappresentazione architettonica del dettaglio costruttivo. Quest'ultimo si pone come lo strumento fondamentale sia per l'analisi della struttura linguistica degli artefatti, sia per il trasferimento inequivoco dei dati di cultura materiale provenienti dalla tecnologia esecutiva. II disegno di dettaglio costruttivo compone una sintesi di duplice natura: oggettiva e interpretativa; esso è la documentazione rigorosa di quanto è visibile ma è completato, negli aspetti relazionali con l'intorno e per quanto non è visibile, dell'interpretazione progettuale dell' intero contesto, controllato con metodo filologico. In concreto: I'idea architettonica è ricomposta sapientemente sulla base del bilanciamento tra evidenze archeologiche e oppor- tunità architettoniche. II disegno è l'espressione preminente e continuistica della cultura storiografica italiana che ha le sue origini negli studi archeologici rinascimentali: si guardi per questo ai rilievi di Giovanni Antonio Dosio (San Gimignano, 1533-Caserta, 1611) (Dosio e Cavalieri, 1569) ${ }^{11}$ o di Pirro Ligorio (Napoli, 1513-Ferrara, 30 ottobre 1583)12 per citare solo alcuni dei Maestri. Si tratta di disegni redatti per comprendere e acquisire il linguaggio classico attraverso la documentazione e l'analisi di leggi compositive, riferimenti culturali ed espedienti costruttivi dimostrati dalle architetture antiche ancora superstiti; il bagaglio d'informazioni ricavato da queste osservazioni alimentava l'esercizio della concinnitas nella progettazione dei necessari interventi di adeguamento all'uso contemporaneo delle architetture del passato. Sin da allora l'interesse antiquario è stato coltivato attraverso lo studio di fonti dirette e fonti indirette accomunate, nei casi più fortunati, ancora dall'uso del disegno: alla produzione di estesi re- 
pertori di rilievi critici, quindi, si accompagnavano i titoli della trattatistica della tradizione vitruviana, particolarmente vitale sino gli albori del XIX secolo. Tra questi è utile rammentare, ai nostri fini, il trattato di Giovanni Antonio Rusconi ${ }^{13}$ e quello di Gherardo Spini ${ }^{14}$ : da entrambi provengono interessantissime ricostruzioni progettuali del processo tecnico di formazione dell'espressione architettonica nell'antichità, attraverso lo studio della natura funzionale ed estetica della costruzione dell'organismo architettonico e degli ordini che lo qualificano. L'argomento è dimostrato attraverso argute descrizioni grafiche dei luoghi comuni della tradizione architettonica, come la genesi tecnologica del templio classico da intendersi come trascrizione in pietra dell'archetipo ligneo. La cultura storicistica descritta ha influito anche sulla pratica del restauro archeologico più maturo, tra il XIX e il XX secolo: si pensi all'attività di Italo Gismondi (Roma, 1887-Roma, 1974) in ambito di documentazione e restauro, con le restituzioni grafiche delle rovine archeologiche ostiensi e la loro coerente ricomposizione materiale ${ }^{15}$, giustificata dal metodo storico-critico di Giuseppe Fiorelli (Napoli, 1823-Napoli, 1896) ${ }^{16}$; sulla base della medesima cultura storicistica, inoltre, si è inteso delineare la singolare specificità della tradizione costruttiva mediterranea di ascendenza romana: valga l'esempio di Gustavo Giovannoni (Roma, 1873-Roma, 1947) chiamato a comporre le note d'italianistica alla ristampa novecentesca dell'edizione italiana dell'imponente trattato mitteleuropeo di costruzioni, opera di Gustavo Adolfo Breymann (Blankenburg, 1807-Stuttgart, 1859) ${ }^{17}$. La linea scientifica descritta si protrae sino a oggi dando vita agli strumenti essenziali e al metodo del «restauro per la valorizzazione» (Pugliano, 2016: 88-92): ne sono espressioni contemporanee i Manuali del Recupero, ovvero raccolte di rilievi critici di manufatti architettonici utili a documentare i lessici costruttivi caratteristici dei diversi luoghi attraverso realia selezionati in base alla loro eloquenza tipologica. I Manuali del Recupero sono nati in Italia alla metà degli anni Ottanta del secolo scorso, grazie a una felice intuizione di Paolo Marconi (Roma, 1933-Roma, 2013) con gli studi sui centri storici di Città di Castello, Roma e Palermo (Pugliano, 2012) e, successivamente, sono divenuti il metodo consueto di approccio allo studio dell'ambiente storico antropizzato; essi hanno ispirato molte esperienze analoghe, anche internazionali, legate alla comune volontà di conservare e valorizzare la memoria fisica delle più significative stratificazioni della cultura urbana ed ediliza ${ }^{18}$.

\section{Un'applicazione in corso: il caso di Tivoli, per} concludere

In applicazione dei principi descritti è in corso una sperimentazione di didattica e di ricerca integrate presso il Laboratorio di restauro architettonico dell'Università Roma Tre, neIl'ambito delle attività legate alla sua Terza Missione. L'attività scientifica è volta al censimento, alla caratterizzazione e al progetto di restauro e valorizzazione di selezionate componenti urbane del centro storico di Tivoli ai fini della fruizione turistica di qualità. In particolare, attualmente, si stanno svolgendo studi storici e tipologici preliminari alla formazione di un Manuale del Recupero; il progetto si giova della realizzazione di una piattaforma digitale da destinare al governo delle iniziative sul territorio e al supporto scientifico della fruizione, tanto diretta quanto virtuale, del paesaggio culturale.

\section{Caratteristiche del sito e motivi dinteresse}

II tessuto urbano storico di Tivoli è particolarmente ricco di possibilità operative, ed esprime un carattere comune a molte delle città laziali di origine antica. Tibur nasce, come Roma del resto, dal sinecismo di pagi e si consolida con la presenza di popoli italici di origine greca (Siculi). L'insediamento arcaico sorse in posizione strategica, sulla riva sinistra dell'Aniene, plausibilmente in corrispondenza dell'attuale Contrada San Paolo, tra il IX e il X secolo a.C. a presidio dell'unico solco vallivo del massiccio montuoso dei monti Tiburtini. Esso fu un crocevia di popoli e commerci; si oppose stenuamente a Roma e nel IV a.C. finalmente, venne annessa alla giurisdizione romana. La fase romana della città fu importantissima e fondativa; essa ha dato luogo a presenze monumentali di singolare rilevanza, architettonica e paesaggistica insieme: si pensi al complesso del Santuario di Ercole Vincitore del II secolo a.C, plausibilmente su preesistenze, o alla villa di Adriano del II d.C., che hanno intercettato l'interesse archeologico degli stessi antiquari rinascimentali che ne trassero feconda ispirazione per gli interventi del nuovo: la realizzazione di Villa d'Este, sotto il governatorato del cardinale ferrarese Ippolito II, vide protagonista proprio Pirro Ligorio, peraltro impegnato nello scavo archeologico di Villa Adriana e nello studio della cosidetta «villa di Augusto» (Ten, 2013: 233-246). Le molte residenze patrizie romane di età tardo-repubblicana dettero vita 
a un contesto paesaggistico singolare, durevole e in qualche misura ancora vitale, come nel caso della villa di Manlio Vopisco, sulla quale è innestata l'ottocentesca villa Gregoriana, o della stessa Villa d'Este il cui Palazzo risulta, da scavi recenti, inglobare tanto una villa romana quanto estesi brani di un convento medioevale, francescano, ritenuto perduto (Fratini e Moricone, 2013: 247-268). La fase medioevale vide Tivoli nel ruolo di sede vescovile, sin dal IV secolo; ruolo che si affievolì nella fase comunale per consolidarsi più tardi, come testimonia l'edificazione della Rocca Pia nel XV secolo, e accrescersi con l'avvento del governatorato degli estensi. La fase medioevale produsse il verificarsi di processi di trasformazione urbana canonici: essa ha consolidato il codice genetico dell'antico tessuto edilizio, trasferendo al futuro la logica dell'aggregazione proprietaria mediata dagli ambiti che scandiscono i perimetri di recinti residenziali riempiti nel tempo da edifici di dimensioni ricorrenti e comuni a quelle delle antiche tabernae. II Medioevo, in più, ha prodotto il consumo della quantità monumentale che è stata metabolizzata, in sostanza attualizzata, attraverso processi ricorrenti di riuso e occupazione. Nella città, l'antichità e le diverse e successive contemporaneità, quindi, hanno convissuto dando luogo a peculiari processi di metamorfosi. Notevoli e ben conservati esempi di permanenza dell'antico sono i volumi sostruttivi e le presenze ipogee di carattere edilizio e infrastrutturale che risultano ben documentati da efficacissime e puntuali campagne archeologiche che si pongono anch'esse in una felice continuità concettuale con le indagini antiquarie Cinquecentesche (Giuliani, 1970).

\section{La proposta progettuale}

La città antica, o almeno la sua memoria concreta, vive dunque estesamente nella città attuale determinandone l'assetto degli edifici e degli spazi urbani. Le evidenze archeologiche cittadine sono solo la parte emergente di un ricco tessuto di relazioni che merita di essere indagato e portato a conoscenza di un pubblico sufficientemente interessato e colto, attraverso selezionate occasioni d'informazione e conoscenza diretta. La valorizzazione, a tal fine, deve riguardare gli attrattori materiali e immateriali: tra i primi la struttura urbana e la tipologia edilizia, con i loro processi di sedimentazione concettuale e di trasformazione materiale tipici e ricorrenti come i processi di accrescimento dei volumi residenziali per taberni- zzazione e il riuso, strumentale alle trasformazioni edilizie, dei lacerti materiali antichi [5]. Tra i secondi: la cultura materiale del luogo, documentata con tavole grafiche nelle quali si opera la ricognizione sul lessico costruttivo locale, per porre le basi per del restauro architettonico e urbano, analizzando e descrivendo sia le concezioni architettoniche dei diversi tipi edilizi d'interesse storico [6], sia la regola dell'arte delle componenti costruttive: murature, tetti, portali e portoni, cornici di finestre, comignoli, per suggerire modelli di comportamento tecnico utili a sanare, attraverso interventi di natura filologica, le debolezze intrinseche agli organismi analizzati [7].

Nel processo di affinamento delle conoscenze sulla città vanno approfondite e poste a sistema, in una piattaforma digitale, le informazioni relative alle reti infrastrutturali antiche e moderne, con i loro lacerti materiali agli artefatti di valore [8]. La piattaforma digitale gestisce informazioni riferite gli assi stradali permanenti, ai tipi edilizi ricorrenti, alle presenze archeologiche e a quant'altro. L'incrocio di dati topografici e architettonici suggerisce interessanti temi conoscitivi da sviluppare: si pensi al sistema idrico cittadino composto di condotti antichi e moderni che è utile porre in relazione alle architetture che da esso vengono alimentate e in particolare al repertorio delle fontane urbane estensi. Queste ultime, sovente misconosciute, rappresentano un interessante documento, nella loro distribuzione cittadina, delle aree di sviluppo e trasformazione edilizia considerate dall'urbanistica estense.

Con queste attività inoltre, è possibile indagare lo stretto rapporto tra edilizia storica emergente e il sostrato archeologico genetico, rintracciando luoghi e condizioni su cui intervenire con una rete di programmi di valorizzazione puntuali così da comporre in città un interessantissimo contesto museale diffuso, concepito su un doppio registro di attrattori, in superficie e ipogei, che sia utile a unire percorsi archeologici e percorsi moderni e contemporanei.

In questo modello di offerta museale, la città di Tivoli, risanata nella compagine materiale, potrebbe essere fruita nella sua interezza, offrendo ai visitatori la massima densità di dati conoscitivi di carattere locale e relazionale. Pertanto: gli interventi di restauro urbano e architettonico che la locale municipalità intenderà avviare e sostenere, sulla base dello studio descritto, dovranno introdurre maggiori livelli di qualità ambientale nel tessuto connettivo dei monumenti archeologici e paesaggistici, contribuendo all'attrattività complessiva del sito [9]. Si tratta di ricostruire, in un insieme composito, il sistema 

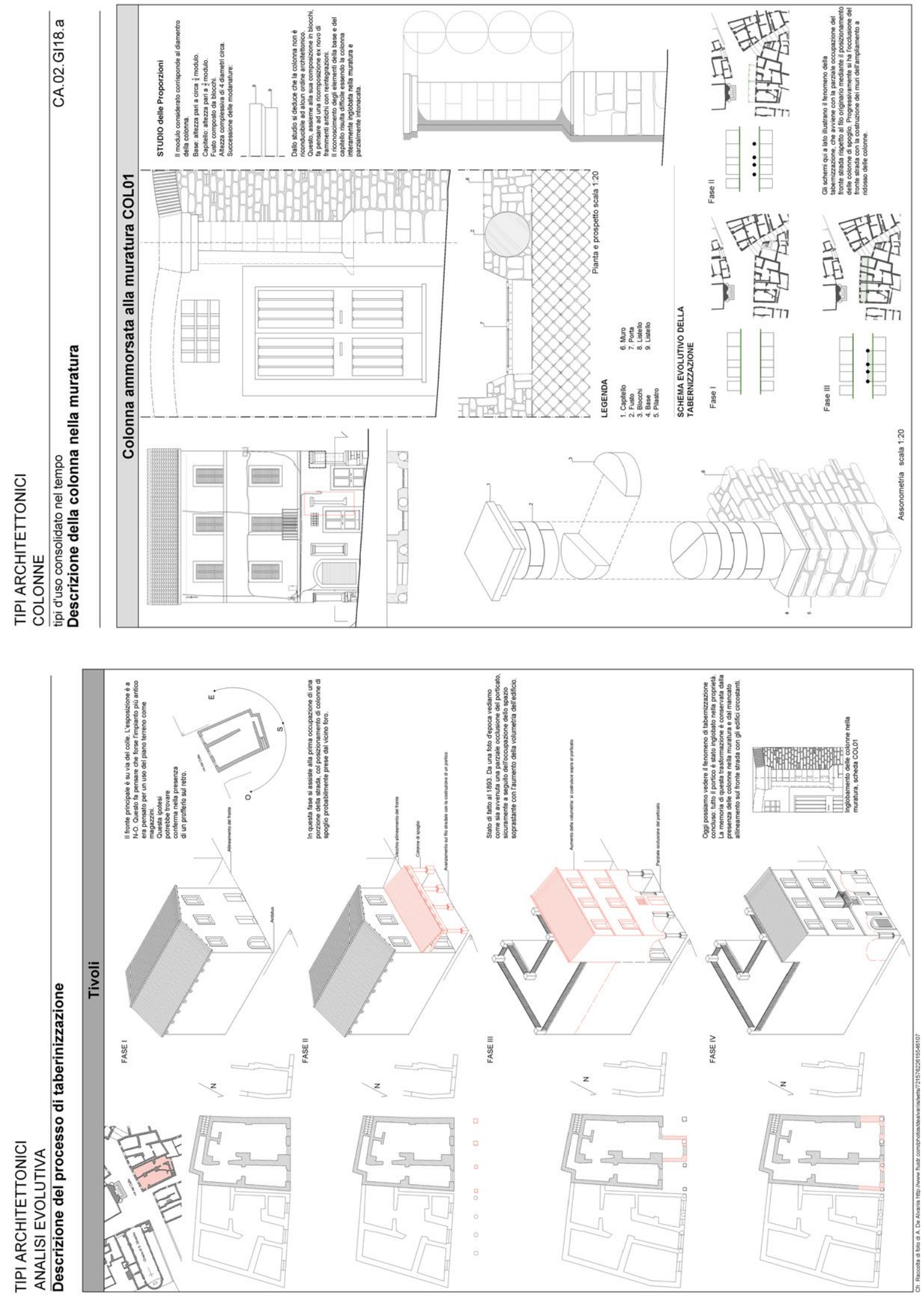

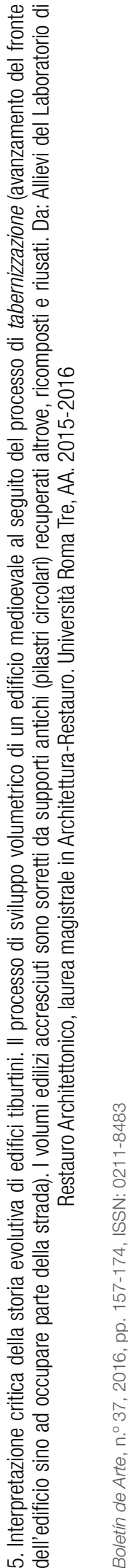



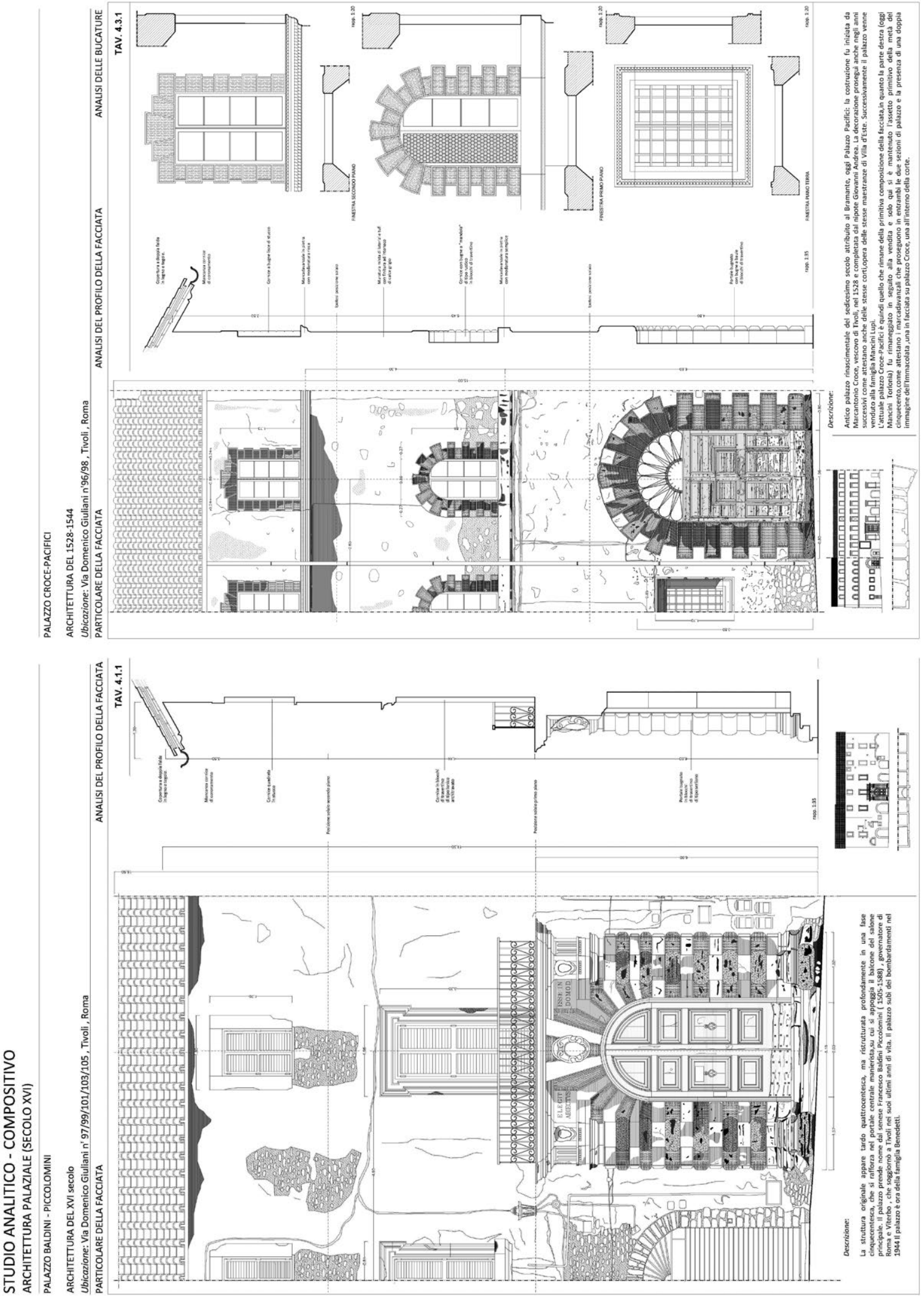


\section{ESEMPI DI TABERNIZZAZIONE SINTESI PROGETTUALE}

\section{Ricomposizione formale ed architettonica - Restauro degli elementi di pregio}

Scheda tecnica n.3

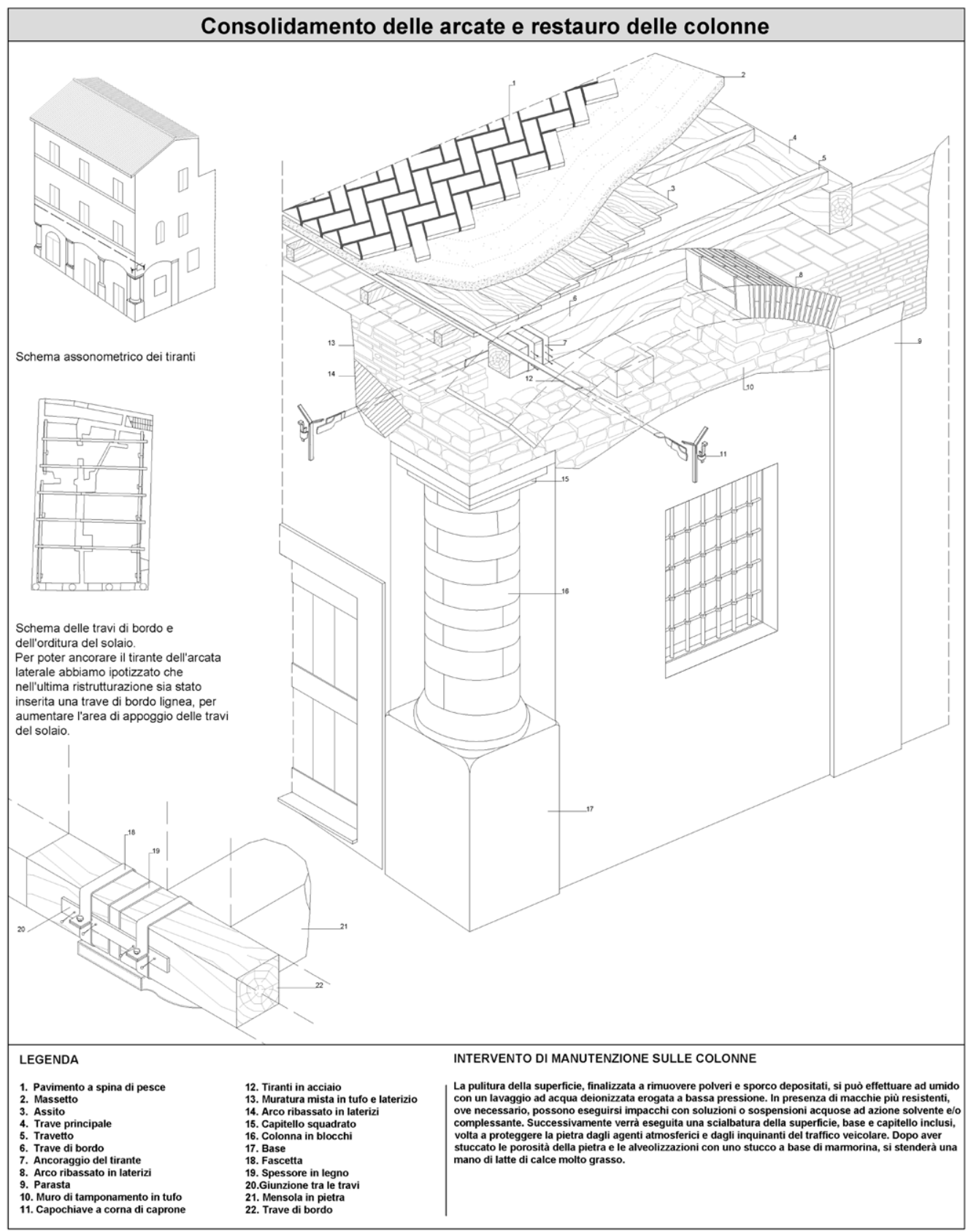

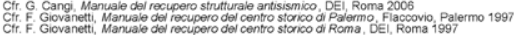

7. Progetto di restauro filologico di un organismo architettonico stratificato contenente lacerti archeologici di riuso. Rinnovo delle pavimentazioni e recupero del solaio in legno; posa in opera di incatenamenti in legno connessi alla struttura del solaio, per il miglioramento della resistenza sismica dell'edificio. Da: Allievi del Laboratorio di Restauro Architettonico, laurea magistrale in Architettura-Restauro. Università Roma Tre AA. $2015-2016$ 

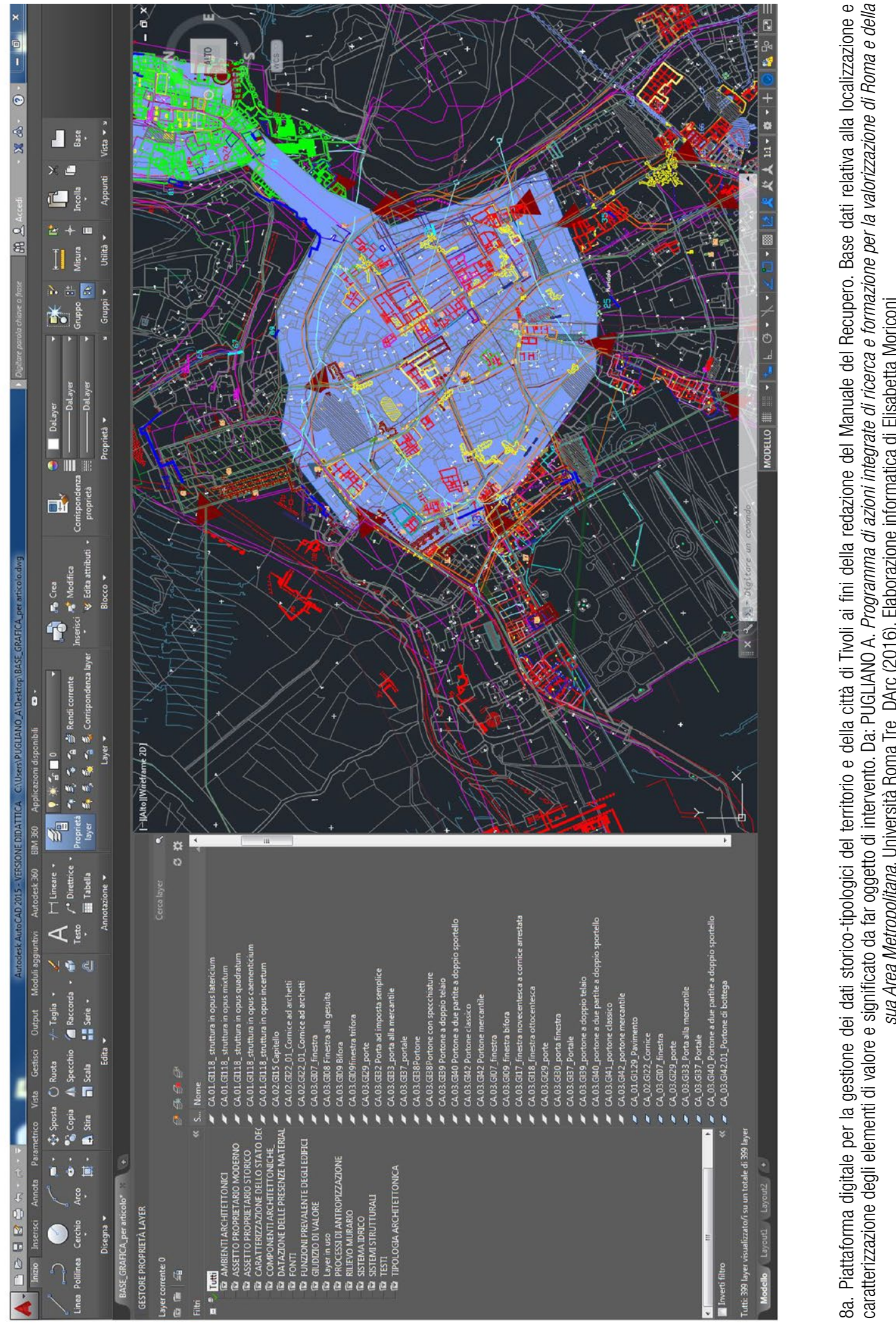

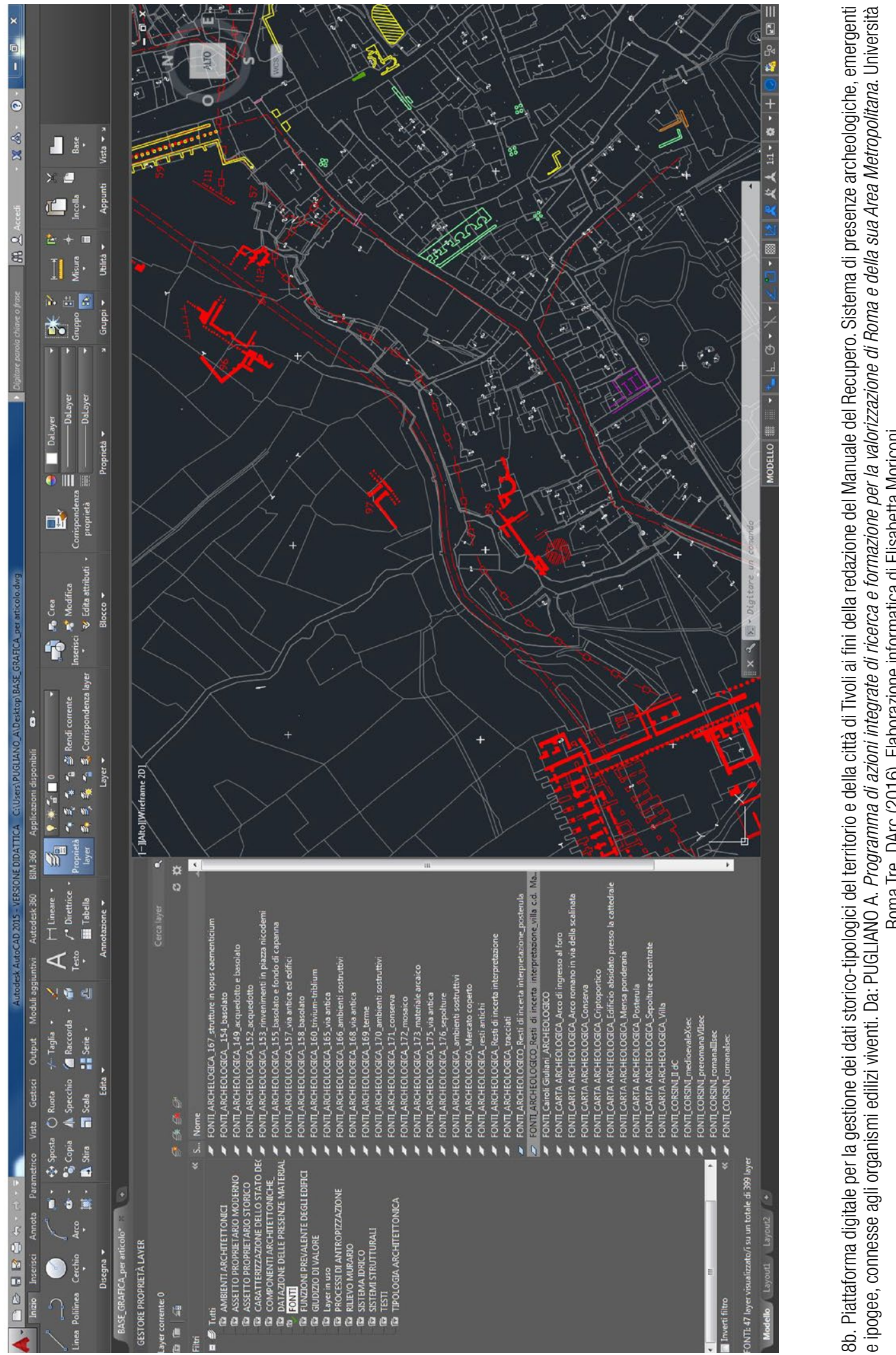


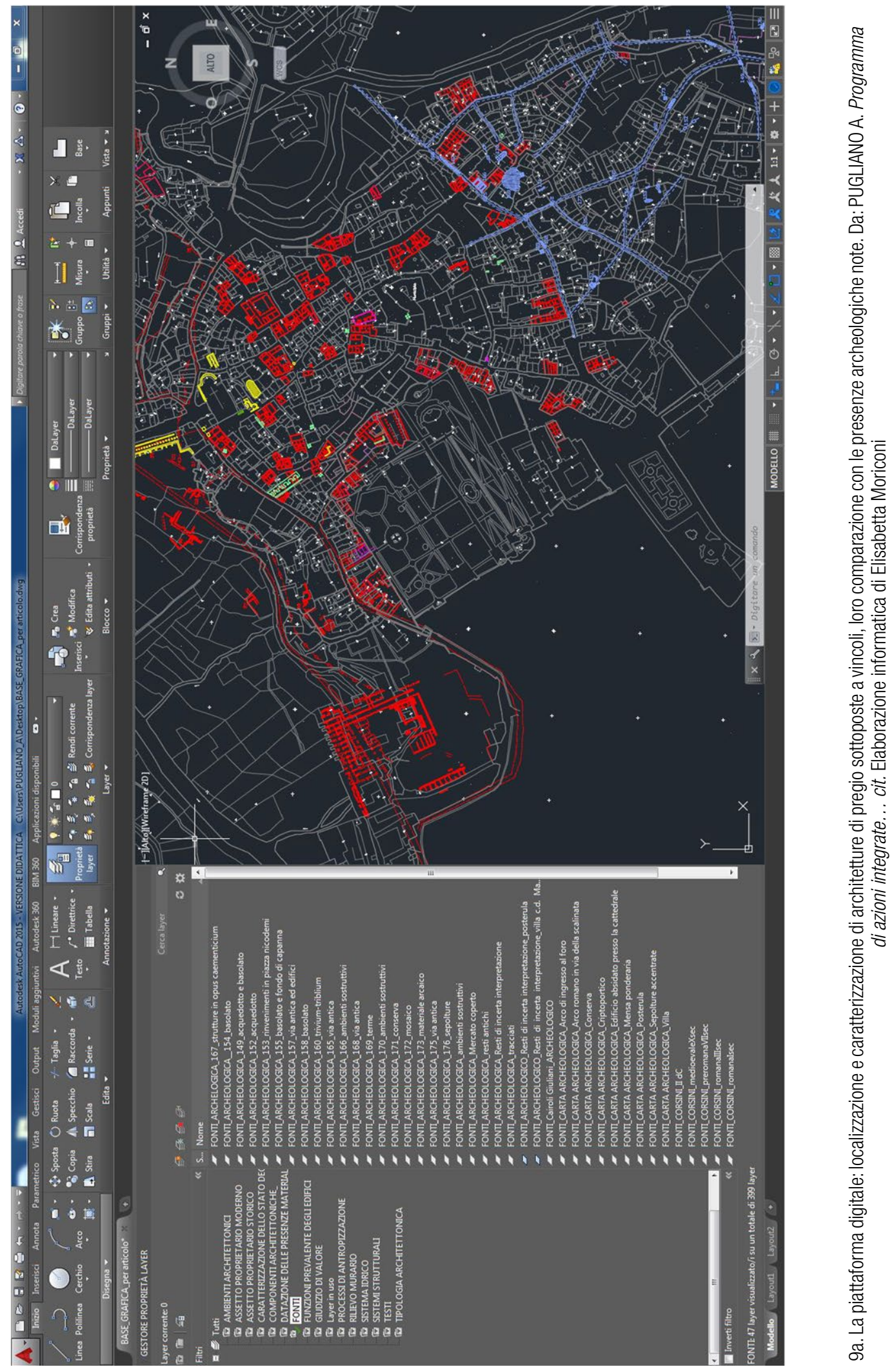




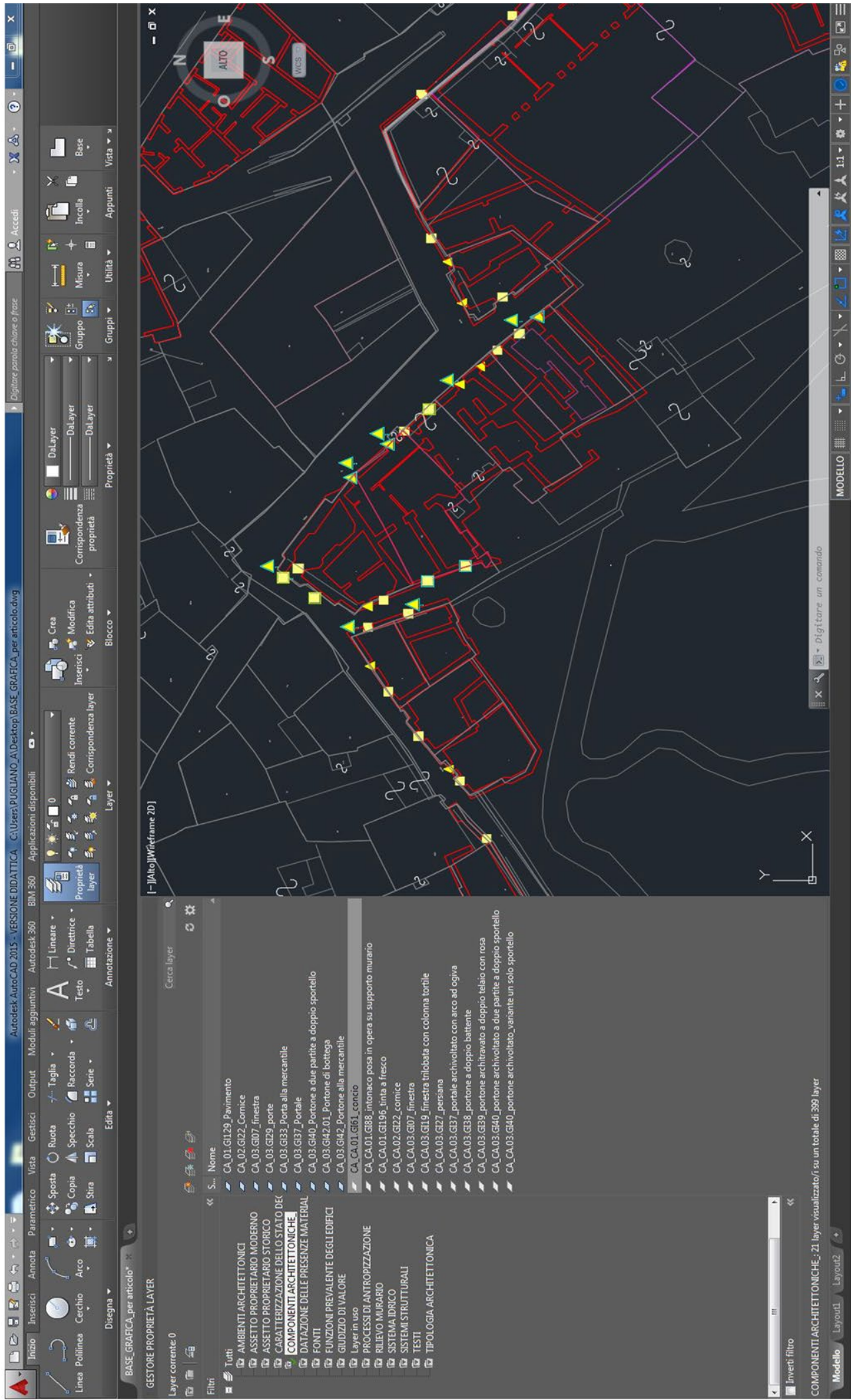

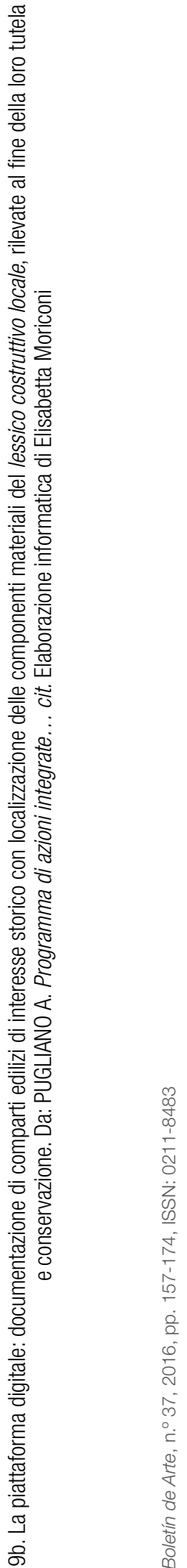



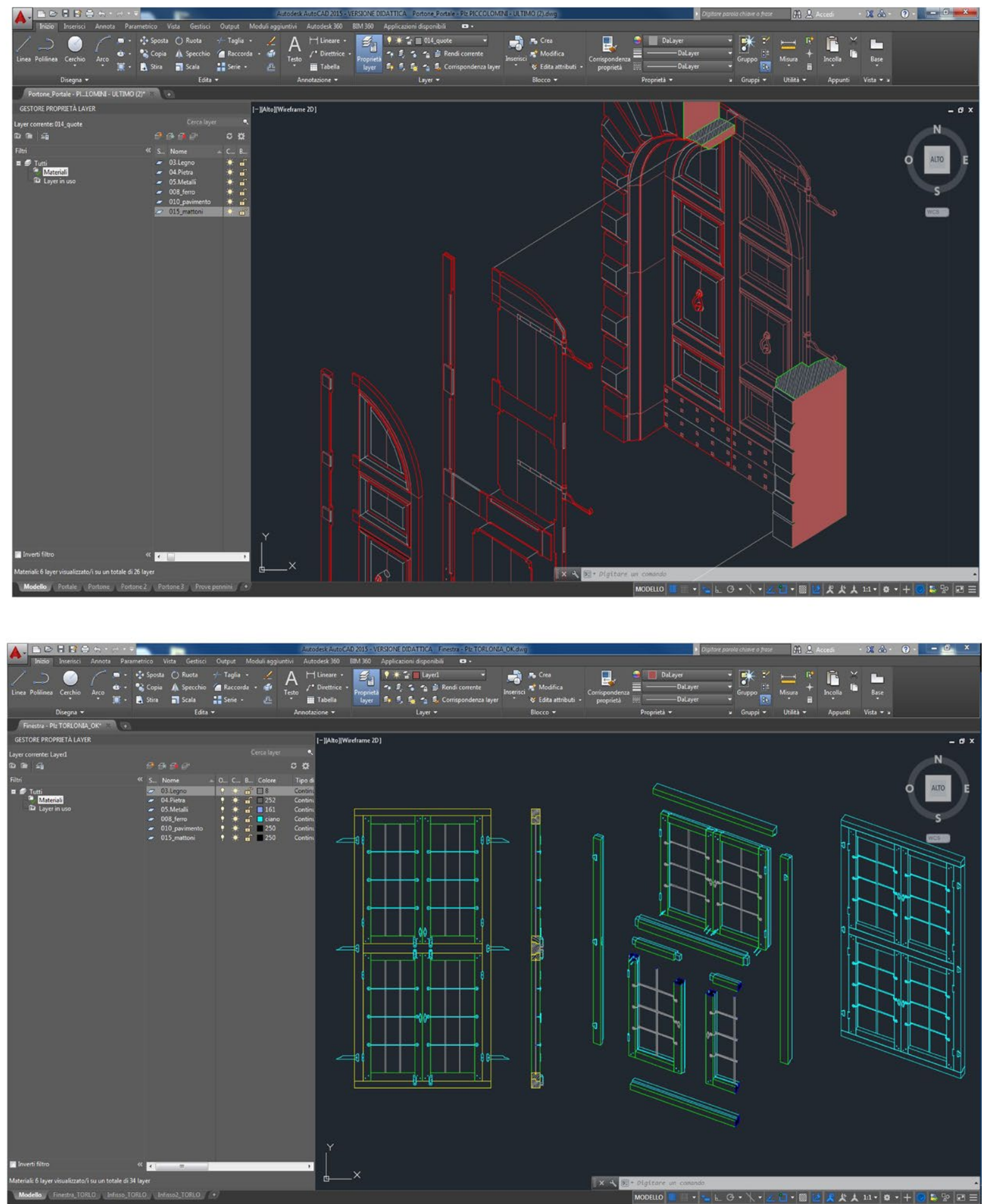

10. La piattaforma digitale: elaborati di dettaglio costruttivo per la descrizione architettonica e tecnologica delle componenti materiali del lessico costruttivo locale. I disegni hanno la qualità del progetto architettonico esecutivo per costituire il riferimento del restauro filologico. Disegno esecutivo di un portale cinquecentesco lapideo completo del portone ligneo (sopra), e di un infisso di finestra rinascimentale, in legno e vetro, con legature in piombo (sotto). Da: PUGLIANO A. Programma di azioni integrate... cit. Elaborazione informatica di Elisabetta Moriconi 
delle presenze di valore per ospitare anche le architetture non monumentali ma d'interesse storico che attualmente sono sottoutilizzate e a rischio di perdita [10]. La costruzione del paesaggio urbano di Tivoli può quindi fornire innegabili vantaggi in termini di valorizzazione dell'economia locale se, interessando la rendita di posizione della proprietà edilizia di base, contribuirà a invertire la tendenza al degrado e abbandono che, in un recente passato, la ricostruzione affrettata e irrazionale dei danni bellici e la successiva deindustrializzazione ha condotto Tivoli alla marginalità attuale, degradandola al ruolo di periferia romana, priva di valore, che necessita di un nuovo e razionale rapporto con il paesaggio culturale cui appartiene.

Note

1 REPUBBLICA ITALIANA. Decreto Legislativo, 22 gennaio 2004, n. 42 recante il «Codice dei beni culturali e del paesaggio» ai sensi dell'articolo 10 della legge 6 luglio 2002, n. 137. Parte Prima. Disposizioni generali. Art. 2-Patrimonio Culturale, commi 1-4.

2 CONSIGLIO D'EUROPA. Comitato dei Ministri della Cultura e dell'Ambiente. Convenzione Europea del Paesaggio. Documento sottoscritto a Firenze il 20 ottobre 2000 e ratificato dal Governo Italiano nel 2006

3 Le premesse alla Convenzione Europea del Paesaggio sono: «la Convenzione relativa alla conservazione della vita selvatica e dell'ambiente naturale d'Europa (Berna, 19 settembre 1979), la Convenzione per la salvaguardia del patrimonio architettonico d'Europa (Granada, 3 ottobre 1985), la Convenzione europea per la tutela del patrimonio archeologico (rivista) (La Valletta, 16 gennaio 1992), la Convenzione-quadro europea sulla cooperazione transfrontaliera delle collettività o autorità territoriali (Madrid, 21 maggio 1980) e i suoi protocolli addizionali, la Carta europea dell'autonomia locale (Strasburgo, 15 ottobre 1985), la Convenzione sulla biodiversità (Rio, 5 giugno 1992), la Convenzione sulla tutela del patrimonio mondiale, culturale e naturale (Parigi, 16 novembre 1972) e la Convenzione relativa all'accesso all'informazione, alla partecipazione del pubblico al processo decisionale e all'accesso alla giustizia in materia ambientale (Aarhus, 25 giugno 1998)». cfr. Convenzione Europea del Paesaggio. «Preambolo».

4 CONSIGLIO D'EUROPA. Convenzione Quadro sul Valore del Patrimonio Culturale per la Società, cosiddetta "Convenzione di Faro", aperta alla firma degli stati membri del Consiglio d'Europa a Faro, in Portogallo, il 27 ottobre 2005 ed entrata in vigore in Italia dal giugno del 2011.

5 Convenzione di Faro. «Preambolo e Parte I: Obiettivi, definizioni e principi. Articolo 1-Obiettivi della Convenzione».

6 Convenzione di Faro. «Parte I: Obiettivi, definizioni e principi. Articolo 1-Obiettivi della Convenzione. Articolo 2-Definizioni».

7 REGNO D'ITALIA. Legge n. 778 dell'11 giugno 1921 Per la tutela delle bellezze naturali e degli immobili di particolare interesse storico.

8 Convenzione Europea del Paesaggio. «Capitolo I-Disposizioni Generali -Articolo 1-Iniziative di qualità-Definizioni».

9 Convenzione di Faro. «Parte I: Obiettivi, definizioni e principi. Articolo 4 -Diritti e responsabilità concernenti I'eredità culturale-Parte II: II contributo dell'eredità culturale alla società e allo sviluppo umano. Articolo 7-Eredità culturale e dialogo. Articolo 8-Ambiente, eredità e qualità della vita. Parte III: Responsabilità condivisa nei confronti dell'eredità culturale e partecipazione del pubblico. Articolo 13-Eredità culturale e conoscenza».

10 Convenzione di Faro. «Parte II: II contributo dell'eredità culturale alla società e allo sviluppo umano. Articolo 9-Uso sostenibile dell'eredità culturale».

11 E per un sintetico approfondimento sull'ambiente degli studi antiquari di Dosio nella Roma del XVI secolo: CARRARA, 2009: 151-160.

12 LIGORIO Pirro, Libro di M. Pyrrho Ligori Napolitano delle antichità di Roma, nel quale si tratta de' circi, theatri e anfitheatri, con le Paradosse del medesimo auttore,-quai confutano la commune opinione sopra varii luoghi della città di Roma. Venezia. Per Michele Tramezino. 1553. LIGORIO Pirro, Il libro delle antichità, manoscritto, cod. 1129, Parigi, Biblioteca Nazionale di Francia. LIGORIO Pirro, XXX Libri delle Antichità, manoscritti, Torino, Archivio di Stato.

13 RUSCONI Giovanni Antonio, Della Architettura di Gio: Antonio Rusconi. Con Centosessanta Figure Disegnate dal medesimo. Secondo i precetti di Vitruvio [...]. Libri Dieci. In Venetia, Appresso i Gioliti MDXC.

14 SPINI Gherardo, I tre primi libri, Venezia, 1568/69, Biblioteca Marciana, ms. It. IV.

15 Per la conoscenza dell'attività di Italo Gismondi ai fini della valorizzazione delle architetture archeologiche si guardi a FILIPPI, 2007.

16 Per gli approfondimenti circa il metodo storico critico di Giuseppe Fiorelli e circa il valore operativo che viene comunemente attribuito a tale metodo si guardi a MARCONI, 2003.

17 BREYMANN, Gustavo Adolfo, Trattato Generale di Costruzioni civili con cenni speciali intorno alle costruzioni grandiose. Vol I, Costruzioni in pietra artificiale, Milano, Francesco Vallardi Editore, s.d. (1885-1926).

18 La più recente in ordine di tempo tra quelle note a chi scrive è: MILETO e VEGAS, 2016.

\section{Bibliografia}

BREYMANN, Gustavo Adolfo (1885-1926), Trattato Generale di Costruzioni civili con cenni speciali intorno alle costruzioni grandiose. Vol I, Costruzioni in pietra artificiale, Francesco Vallardi Editore, s.d., Milano.

CARRARA, Eliana (2009), «Giovanni Antonio Dosio e Giovanni Battista Naldini: pratica del disegno e studio dell'antico nella Roma del settimo decennio del Cinquecento", in DI TEODORO, Francesco Paolo (a cura), Saggi di Letteratura architettonica, da Vitruvio a Winckelmann, vol. I, Firenze, Leo S. Olschki Editori, Firenze, pp. 151-160. 
COGOTTI, Marina (2015), Tivoli. Paesaggio del Gran Tour. Contributo alla conoscenza e al recupero del paesaggio tiburtino, De Luca Editori d'Arte, Roma.

CONSIGLIO D'EUROPA. Comitato dei Ministri della Cultura e dell'Ambiente. Convenzione Europea del Paesaggio. Documento sottoscritto a Firenze il 20 ottobre 2000 e ratificato dal Governo Italiano nel 2006.

- Convenzione Quadro sul Valore del Patrimonio Culturale per la Società, cosiddetta «Convenzione di Faro», aperta alla firma degli stati membri del Consiglio d'Europa a Faro, in Portogallo, il 27 ottobre 2005 ed entrata in vigore in Italia dal giugno del 2011.

Convenzione di Faro. «Preambolo e Parte I: Obiettivi, definizioni e principi. Articolo 1-Obiettivi della Convenzione».

— «Parte I: Obiettivi, definizioni e principi. Articolo 1 -Obiettivi della Convenzione. Articolo 2-Definizioni».

— «Parte I: Obiettivi, definizioni e principi. Articolo 4 -Diritti e responsabilità concernenti l'eredità culturale-Parte II: Il contributo dell'eredità culturale alla società e allo sviluppo umano. Articolo 7-Eredità culturale e dialogo. Articolo 8-Ambiente, eredità e qualità della vita. Parte III: Responsabilità condivisa nei confronti dell'eredità culturale e partecipazione del pubblico. Articolo 13-Eredità culturale e conoscenza».

— «Parte II: II contributo dell'eredità culturale alla società e allo sviluppo umano. Articolo 9-Uso sostenibile dell'eredità culturale.»

Convenzione Europea del Paesaggio. «Capitolo I-Disposizioni Generali -Articolo 1-Iniziative di qualità-Definizioni».

DOSIO G. A.; CAVALIERI G. B. (1569), Urbis Romae. Aedificiorum Illustrium quae supersunt reliquiae, Roma.

FRATINI, Giulio; MORICONI, Francesco (2013), «ll cantiere di Villa d'Este. Preesistenze, condizionamenti e modifiche in corso d'opera», in FIORE, Francesco Paolo e COGOTTI, Marina, Ippolito // d'Este. Cardinale, Principe, Mecenate, Atti del Convegno, De Luca, Roma, pp. 247-268.

FILIPPI, F. (2007), Ricostruire l'Antico prima del virtuale. Italo Gismondi. Un architetto per l'archeologia (1887-1974), Quasar, Roma. GIULIANI, Fulvio Cairoli (1970), Forma Italiae. Regio I, Volumen Septimum. Tibur. Pars Prima, De Luca Editore, Roma.

GOMBRICH, E. H. (1977), La storia dell'arte raccontata, Einaudi, Milano.

LIGORIO, Pirro (1553), Libro di M. Pyrrho Ligori Napolitano delle antichità di Roma, nel quale si tratta de' circi, theatri e anfitheatri, con le Paradosse del medesimo auttore,-quai confutano la commune opinione sopra varii luoghi della città di Roma. Venezia. Per Michele Tramezino.

- Il libro delle antichità, manoscritto, cod. 1129, Parigi, Biblioteca Nazionale di Francia.

- XXX Libri delle Antichità, manoscritti, Torino, Archivio di Stato.

MANIGLIO CALCAGNO, Annamaria (2015), Per un paesaggio di qualità. Dialogo su inadempienze e ritardi nell'attuazione della Convenzione Europea, Franco Angeli, Milano.

MARCONI, P. (2003), II restauro e l'architetto, Marsilio, Venecia.

MILETO, C. y VEGAS, F. (2016), Centro Histórico de Valencia. Ocho siglos de arquitectura residencial. 2 voll., TC Cuadernos, Valencia. NIETZSCHE, F. W. (1992), Aurora, Adelphi Edizioni, Milano.

TEN, Alessandra (2013), «Pirro Ligorio, Villa d'Este e le antichità tiburtine: interferenze tra architettura e archeologia», in FIORE Francesco Paolo e COGOTTI, Marina, Ippolito II d'Este. Cardinale, Principe, Mecenate, De Luca, Roma, pp. 233-246.

TEODORO, Francesco Paolo (a cura) (2009), Saggi di Letteratura architettonica, da Vitruvio a Winkelmann, vol I, Leo S. Olschki Editori, Firenze, pp. 151-160.

PUGLIANO, Antonio (2012), «La conservación de la arquitectura y los espacios culturales a principios del siglo XXI. Nuevos instrumentos para el reconocimiento del valor y para la restauración del Patrimonio Cultural italiano». En: <http://www.uco.es/arte/revista/index. html.10/4/2016>.

- (2016), «Paolo Marconi e il restauro per la valorizzazione», Ricerche di Storia dell'Arte, nn. 116-117, Carocci, Roma pp. 88-92. REGNO D'ITALIA. Legge n. 778 dell'11 giugno 1921 Per la tutela delle bellezze naturali e degli immobili di particolare interesse storico.

REPUBBLICA ITALIANA. Decreto Legislativo, 22 gennaio 2004, n. 42 recante il «Codice dei beni culturali e del paesaggio» ai sensi dell'articolo 10 della legge 6 luglio 2002, n. 137. Parte Prima. Disposizioni generali. Art. 2-Patrimonio Culturale, commi 1-4.

RUSCONI, Giovanni Antonio, Della Architettura di Gio: Antonio Rusconi. Con Centosessanta Figure Disegnate dal medesimo. Secondo i precetti di Vitruvio [...]. Libri Dieci. In Venetia, Appresso i Gioliti MDXC.

SPINI, Gherardo, I tre primi libri, Venezia, 1568/69, Biblioteca Marciana, ms. It. IV. 